\title{
Efetividade da intervenção breve para o uso abusivo de álcool na atenção primária: revisão sistemática
}

\author{
Effectiveness of the brief intervention for the use of abusive alcohol in the primary: systematic review
}

Eficacia de la intervención breve para el uso de abusivo de alcohol en la atención primaria: revisión sistemática

\begin{abstract}
Maria Odete Pereira', Bárbara Marques Anginoni", Natany da Costa Ferreira", Márcia Aparecida Ferreira de Oliveira"', Divane de Vargas"'I, Luciana de Almeida Colvero"'I

' Universidade de São Paulo, Escola de Enfermagem, Programa de Pós-Graduação em Enfermagem (Pós-doutoranda), Grupo de Estudos Relacionados ao Uso Prejudicial do Álcool e Outras Drogas. São Paulo-SP, Brasil.

"Universidade de São Paulo, Escola de Enfermagem (Graduanda), Grupo de Estudos Relacionados ao Uso Prejudicial do Álcool e Outras Drogas. São Paulo-SP, Brasil.

I' Universidade de São Paulo, Escola de Enfermagem, Departamento de Enfermagem Materno-Infantil e Psiquiátrica, Grupo de Estudos Relacionados ao Uso Prejudicial do Álcool e Outras Drogas. São Paulo-SP, Brasil.
\end{abstract}

\author{
Submissão: 24-05-2011 Aprovação: 09-04-2013
}

\begin{abstract}
RESUMO
A pesquisa identificou estudos clínicos acerca das intervenções breves para o uso abusivo de álcool e analisou a eficácia dos aconselhamentos para os consumidores. Estudo bibliográfico sistemático que utilizou artigos publicados no período de 1997 a 2010. Os achados mostraram que a estratégia de intervenção breve é efetiva na redução da frequência e quantidade de álcool, com melhores resultados quando aplicada na atenção primária. Todos os profissionais da área da saúde obtêm bons resultados com o manejo dessa terapia. Não foi possível definir se a estratégia é mais eficaz se aplicada em bebedores de uso nocivo ou em crônicos, uma vez que a intervenção depende da mobilização de recursos motivacionais do paciente e de mudanças de atitude relacionadas ao consumo de álcool.

Descritores: Álcool; Intervenção Breve; Atenção Primária; Tratamento, Efetividade.
\end{abstract}

\begin{abstract}
ABTRACT
The research identified clinical studies on the brief interventions for the abusive use of alcohol and analyzed the effectiveness of professional advises proposed to alcohol consumers. The systematic bibliographical study was based on articles published during the period of 1997 to 2010. Brief intervention strategy is effective in the reduction on the frequency and amount of alcohol use, with better results when the strategy is applied to the primary attention situations. All professionals obtained good results using this therapy. It was not possible to determine if the strategy is most effective when applied to harmful drinkers or to chronic drinkers, since the intervention also depends on the patient's availability of motivational resources and on changes in attitude regarding the abusive use of alcohol.
\end{abstract}

Key words: Alcohol; Brief Intervention; Basic Attention; Treatment; Effectiveness.

\section{RESUMEN}

La encuesta identificó estudios clínicos sobre intervenciones breves para el abuso de alcohol y analizado la eficacia de la orientación a los consumidores. Estudio sistemático utilizó la literatura publicada entre 1997 y 2010 . Resultados mostraron que la estrategia de intervención breve es efectiva en la reducción de la frecuencia y la cantidad de alcohol, con mejores resultados cuando se utilizan para primaria. Todas atenciones en el área de la salud han tenido buenos resultados con la gestión de esta terapia. No se puede establecer la estrategia es más eficaz si se aplica en los bebedores de abuso de alcohol o crónica, ya que la intervención depende de la movilización de los cambios motivacionales y actitudinales del paciente relacionados con el consumo de alcohol.

Palabras clave: El Alcohol; Intervenciones Breves; La Atención básica; El Tratamiento; La Eficacia.

\section{AUTOR CORRESPONDENTEＭaria Odete Pereira E-mail: mariaodete@usp.br}




\section{INTRODUÇÃO}

Acredita-se que a bebida alcoólica teve origem na Pré-História, com o aparecimento da agricultura e com a invenção da cerâmica. Somente na Idade Contemporânea é que o uso excessivo de bebida passa a ser visto como uma doença ou desordem. Historicamente, o álcool teve importâncias econômicas, sociais, religiosas e medicamentosas, já que era consumido em todos os segmentos da sociedade ${ }^{(1)}$.

Embora já no final do século XVIII autores americanos e europeus escrevessem acerca do consumo de álcool relacionando-o ao adoecimento, foi no início do século XIX que o modelo biomédico ganhou força, e surgiu a idéia de tratamento para as complicações graves decorrentes do uso crônico, como tentativa de mudar o foco de moralismo e de vergonha que pairava sobre o "bêbado". Até aquele momento todas as políticas públicas existentes relacionadas à temática eram de caráter repressivo e moralizador.

A Associação Americana de Psiquiatria - APA e o Manual Diagnóstico e Estatístico de Desordens Mentais - DSMI incluíram o alcoolismo em sua primeira versão em 1952 e a sua inclusão no CID-8, da Organização Mundial de Saúde - OMS se deu em 1967, já os conceitos de abuso e dependência foram estabelecidos pela OMS em 1977. Os procedimentos dessas duas taxonomias reforçaram o caráter moralizador do alcoolismo, que colocava o beber excessivo habitual como falta de vergonha e um caso de polícia, expressões que perduraram durante muito tempo ${ }^{(2)}$.

São diversas as concepções acerca do conceito de saúde no século XXI, uma vez que a noção de saúde como completo bem-estar físico, psíquico e social, como coloca a OMS é tido como utópico e se contrapõe ao estilo de vida que a maioria das pessoas tem. Nessa perspectiva, a idéia de perfeição e completude nas três dimensões em relação ao estado de saúde coloca quase que a totalidade das pessoas na condição de ausência de saúde ${ }^{(3)}$.

A subjetividade exerce uma influência direta na representação que o conceito saúde tem para as pessoas, uma vez que saúde não possui o mesmo significado para todas elas, pois depende do contexto em que vivem, suas culturas, crenças, experiências, concepções filosóficas e científicas ${ }^{(4)}$.

Nessa óptica, outros conceitos relacionados ao processo saúde-doença são repensados já no século XX, e dentre eles aqueles que dizem respeito ao uso abusivo de álcool e outras drogas, como referido em parágrafo anterior, assim, tornou-se evidente a necessidade de ações de prevenção para o uso de risco de bebidas alcoólicas. Hoje não se pode falar apenas em abstinência como uma política de resolução e tratamento dessa condição, mas deve-se pensar no desenvolvimento de programas que estimulem a competência dos usuários, para promover "resiliência" (cunho do autor) ao uso de álcool, termo que tem sido utilizado para designar a capacidade de resistir às pressões externas e internas que facilitariam o uso de tal substância ${ }^{(5)}$.

Nesse sentido, em 1972, no Canadá, é proposta a Intervenção Breve - IB como abordagem psicoterapêutica para dependentes de álcool por Sanchez-Craig et al.(6). A estratégia consiste na aplicação de quatro sessões focalizadas e simples, no entanto, seus autores observaram uma redução imediata do consumo de álcool em dependentes graves e, consequentemente, uma melhora na saúde, quando comparada a uma amostra semelhante de pacientes sem tratamento.

De uma forma geral, a IB apresenta um enfoque educativo e motivacional, em que o principal objetivo é desencadear a decisão e o comprometimento com a mudança dos pacientes, com a finalidade de reduzir o risco de danos ocasionados pelo consumo exagerado de álcool ${ }^{(7)}$.

O espaço terapêutico de eleição para a intervenção breve é a atenção primária, devido à possibilidade de vínculo entre os usuários e equipe de saúde e efetividade do monitoramento do tratamento.

Alguns autores, embora tenham desenvolvido estudos em serviços emergenciais e ambulatoriais, concluem que a IB na atenção primária é mais efetiva, uma vez que o indivíduo não se encontra em um processo tão avançado de dependência química.

Nessa lógica, os autores do presente estudo se propõem a realizar uma revisão sistemática da literatura, com a finalidade de identificar a produção bibliográfica acerca das intervenções breves realizadas na atenção primária, no Brasil, nos últimos treze anos e que foram divulgadas nas principais bases de dados brasileiras, na área da saúde.

\section{OBJETIVOS}

O presente trabalho objetivou identificar artigos que contemplassem o critério de estudo empírico a respeito do emprego de estratégias de intervenções breves no uso abusivo de álcool; e analisar a eficácia e os impactos dos aconselhamentos propostos aos participantes, nos estudos selecionados.

\section{METODOLOGIA}

Trata-se de um estudo bibliográfico sistemático das publicações divulgadas nas Bases de Dados da Biblioteca Virtual de Saúde - BIREME (Scielo, Lilacs e Medline), no período de 1997 a 2010. A seleção dos textos apoiou-se em descritores previamente determinados: álcool, intervenção breve, atenção primária, tratamento e efetividade. O número de descritores pautou-se nas normas para publicação de periódicos em Enfermagem.

O período foi escolhido devido ao fato de que as primeiras publicações científicas acerca das intervenções breves para o uso abusivo de álcool são datadas a partir da década de 1970 e, produzidas essencialmente naquela época por países desenvolvidos como Estados Unidos, Canadá, Inglaterra e Espanha.

Os critérios de inclusão de artigos que versam a respeito de intervenções breves foram os seguintes: delineamento da pesquisa, com seleção apenas de estudos empíricos; objetivos, métodos e resultados claramente definidos no resumo do artigo; artigos publicados nas línguas portuguesa, inglesa e espanhola; artigos publicados entre os anos de 1997 a 2010.

\section{RESULTADOS}

A busca limitada pelos parâmetros determinados anteriormente resultou em um total de 486 artigos. Dentre esses, os artigos 
referentes a pesquisas de revisão literária, artigos que não abordavam a temática de efetividade das IBs com os usuários dos serviços de saúde (relacionados à capacitação de gestores da saúde, capacitação de agentes comunitários, redução de danos e etc.) e artigos cujos resultados ainda não estavam concluídos quando publicados, foram excluídos. A amostra final foi, portanto, composta por 30 itens, apresentados a seguir na tabela 1.

Dentre os artigos identificados, prevaleceram aqueles em que foram utilizados os descritores tratamento e álcool, com 226 itens, na Scielo, seguidos daqueles em que foram empregados os descritores álcool, intervenção breve, atenção primária e efetividade, com 89 itens, selecionados no Medline.

Tabela 1 - Resultados da busca bibliográfica nas bases de dados da Bireme, entre outubro de 1997 e janeiro de 2010.

\begin{tabular}{l|l|c}
\hline BANCO DE DADOS & DESCRITORES & NÚMERO DE ARTIGOS \\
\hline Lilacs & 1. álcool and etílico and intervenção & 7 \\
\hline & 2. álcool and intervenção and breve & 17 \\
\hline & 3. intervenção breve and atenção primária & 5 \\
\hline & 4. álcool and atenção primária & 41 \\
\hline & 5. alcohol and intervention and brief & 15 \\
\hline & 6. álcool and tratamento breve & 20 \\
\hline Total de artigos após filtragem & 1. álcool and intervenção & $\mathbf{5}$ \\
\hline Scielo & 2. álcool and atenção primária & 44 \\
\hline & 3. intervenção breve and atenção primária & 12 \\
\hline & 4. álcool and intervenção breve & 0 \\
\hline & 5. tratamento and álcool & 10 \\
\hline & 1. alcohol and intervention and brief and primary care and effectiveness & 226 \\
\hline Total de artigos após filtragem & $\mathbf{2}$ \\
\hline Medline & 89 \\
\hline Total de artigos após filtragem & $\mathbf{2 3}$ \\
\hline TOTAL DE ARTIGOS SELECIONADOS & $\mathbf{3 0}$ \\
\hline
\end{tabular}

Quadro 1 - Distribuição dos artigos de revisão bibliográfica publicados nas Bases de dados da Bireme, entre outubro de 1997 e janeiro de 2010, selecionados segundo ano; autor(es) e País de origem.

\begin{tabular}{|c|c|c|}
\hline ANO & AUTORES & LOCAL \\
\hline 2010 & Rubio G e colaboradores & Estados Unidos da América \\
\hline 2009 & Castro LA e colaboradores & Brasil \\
\hline 2009 & Alarcón LF e colaboradores & Colômbia \\
\hline 2009 & Ockene TK e colaboradores & Estados Unidos da América \\
\hline 2009 & Stein LA e colaboradores & Estados Unidos da América \\
\hline 2009 & Schaus JF e colaboradores & Estados Unidos da América \\
\hline 2009 & Tariq L e colaboradores & Estados Unidos da América \\
\hline 2008 & Oliveira MS e colaboradores & Brasil \\
\hline 2008 & Andretta I e colaboradores & Brasil \\
\hline 2008 & Fleming MF e colaboradores & Inglaterra \\
\hline 2008 & Dent AW e colaboradores & Austrália \\
\hline 2008 & Guth S e colaboradores & Estados Unidos da América \\
\hline 2008 & Grothues JN e colaboradores & Irlanda \\
\hline 2007 & Batista AG e colaboradores & Brasil \\
\hline 2007 & Ball AS e colaboradores & Estados Unidos da América \\
\hline 2007 & Brown RL e colaboradores & Inglaterra \\
\hline 2006 & Lock CA e colaboradores & Inglaterra \\
\hline 2005 & Laranjeira R e colaboradores & Brasil \\
\hline 2005 & López-Marina V e colaboradores & Espanha \\
\hline 2005 & Mundt MP e colaboradores & Estados Unidos da América \\
\hline 2004 & De Michele D e colaboradores & Brasil \\
\hline 2003 & Lieber CS e colaboradores & Estados Unidos da América \\
\hline 2003 & Gordon AJ e colaboradores & Estados Unidos da América \\
\hline 2003 & Fernández GJA e colaboradores & Espanha \\
\hline 2001 & Kaner EF e colaboradores & Inglaterra \\
\hline 2001 & Aalto M e colaboradores & Inglaterra \\
\hline 2001 & Aalto M e colaboradores & Estados Unidos da América \\
\hline 2000 & Manwell LB e colaboradores & Estados Unidos da América \\
\hline 1998 & Córdoba R e colaboradores & Inglaterra \\
\hline 1997 & Fernandez SMMI e colaboradores & Espanha \\
\hline Total & 30 & 07 Países \\
\hline
\end{tabular}


Após o processo de seleção dos artigos, procedeu-se à tabulação dos dados encontrados com a discriminação dos seguintes aspectos: ano; autor; país em que trabalho foi realizado; local de aplicação da IB; caracterização da população estudada; uso de teste de rastreamento; e efetividade das IBs, apresentados nos quadros 1, 2 e 3.
Entre os anos de 1997 e 2010 foram publicados 30 artigos, sendo que em 2008 e 2009 foram divulgados seis trabalhos, respectivamente. Observou-se que a partir de 2001, verificou-se a crescente e linear produção de artigos em periódicos, com notável ascendência a partir de 2008.

Os trabalhos foram publicados em sete países, sendo 12

Quadro 2 - Distribuição de artigos selecionados nas Bases de dados da Bireme, entre outubro de 1997 e janeiro de 2010 , segundo local de aplicação; caracterização da população estudada e uso de teste de rastreamento dos estudos de revisão bibliográfica.

\begin{tabular}{|c|c|c|c|}
\hline $\begin{array}{l}\text { Artigo } \\
\text { número de } \\
\text { Referência }\end{array}$ & Local de aplicação & Participantes* & Teste de Rastreamento \\
\hline 08 & Atenção Primária & 15.325 & Não consta \\
\hline 09 & Ambulatório & $71 ;$ homens e mulheres; $18-65$ & Não consta \\
\hline 10 & Não Consta & 180; universitários & CAGE \\
\hline 11 & Atenção Primária & Não consta & Não consta \\
\hline 12 & Hospital & $\begin{array}{l}\text { 444; } 27 \text { anos (média); } 346 \text { (78\%) } \\
\text { homens }\end{array}$ & AUDIT \\
\hline 13 & Atenção Primária & 363; universitários; 189 (52\%) homens & $\begin{array}{l}\text { Rutgers Alcohol Problem Index (RAPI) e } \\
\text { Drinker Inventory of Consequences }\end{array}$ \\
\hline 14 & Atenção Primária & Homens e mulheres; 20-65 anos & AUDIT e Chronic Disease Model (CDM) \\
\hline 15 & Não Consta & 152; homens; $25-60$ anos & Entrevista estruturada e FORM 90 \\
\hline 16 & $\begin{array}{l}\text { Instituições Especializadas no } \\
\text { Comprimento de Medidas } \\
\text { Protetivas a Adolescentes }\end{array}$ & 50; adolescentes; 13 -20 anos & $\begin{array}{l}\text { Entrevista semi-estruturada e } \\
\text { screening toxológico }\end{array}$ \\
\hline 17 & Clínicas Obstétricas & 235; mulheres; 28 anos (média) & $\begin{array}{l}\text { TACE (modificação do CAGE } \\
\text { para ambiente obstétrico) }\end{array}$ \\
\hline 18 & Serviço de Emergência & 468 & Teste de álcool Paddington \\
\hline 19 & Atenção Primária & 326 & Não consta \\
\hline 20 & Ambulatório & 408 & Não consta \\
\hline 21 & Batalhão de Bombeiros Militares & 303; homens; 33,6 anos (média) & AUDIT \\
\hline 22 & Ambulatório & 461 & Não consta \\
\hline 23 & Atenção Primária & $445 ; 199$ homens e 246 mulheres & Não consta \\
\hline 24 & Atenção Primária & 127 homens & Não consta \\
\hline 25 & Ambulatório & $\begin{array}{l}316 ; 202 \text { homens ( } 64 \%) \text { e } 114 \\
\text { mulheres; } 40-50 \text { anos }\end{array}$ & AUDIT \\
\hline 26 & Atenção Primária & $78 ; 18-65$ anos & Não consta \\
\hline 27 & Não Consta & 158; maiores de 65 anos & Não consta \\
\hline 28 & Ambulatório & 108; adolescentes; 10-19 anos & DUSI \\
\hline 29 & Não Consta & 789 & Não consta \\
\hline 30 & Atenção Primária & $45 ; 65$ anos e acima & Não consta \\
\hline 31 & Atenção Primária & 306 & CAGE \\
\hline 32 & Atenção Primária & $\begin{array}{l}\text { 2043; } 16 \text { anos e acima; } 1184 \text { (58\%) } \\
\text { homens }\end{array}$ & AUDIT \\
\hline 33 & Atenção Primária & 296; homens & Não consta \\
\hline 34 & Ambulatório & 118; mulheres & Não consta \\
\hline 35 & Atenção primária & 205; mulheres; 18-40 & Não consta \\
\hline 36 & Atenção primária & 229; homens; 18-65 & CAGE \\
\hline 37 & Atenção primária & $152 ;$ homens & Não consta \\
\hline
\end{tabular}

* número de participantes; gênero; idades e grupos etários 
deles elaborados por pesquisadores dos Estados Unidos da América. No período, o Brasil e a Inglaterra produziram seis trabalhos, cada um, posicionando-se na segunda posição de trabalhos divulgados nas bases de dados da Bireme.

Dos 30 trabalhos que preencheram os critérios estabelecidos para esta pesquisa, 14 foram desenvolvidos em ambientes da atenção primária, e seis em espaços ambulatoriais.

Quanto ao público-alvo, as idades prevalentes entre os participantes foram entre 18 e 65 anos. O público adolescente e universitário que se mostrou representativo, uma vez que quatro artigos publicados enfocaram esse grupo etário. Vale ressaltar que dois artigos tiveram com amostra uma população idosa, na faixa etária de 65 anos e acima.

A respeito do gênero, sete estudos tiveram como amostra o sexo masculino, três o sexo feminino, e catorze se caracterizaram por analisar ambos os sexos.
Em 16 trabalhos os autores não utilizaram instrumentos validados para rastreamento de consumo de álcool. O AUDIT foi empregado para o rastreamento em cinco trabalhos, sendo que em um deles foi associado a outro teste.

As principais estratégias utilizadas pelos pesquisadores foram: aconselhamento breve e entrevista motivacional. De maneira geral, os artigos incluídos no presente estudo pretenderam analisar os efeitos e/ou a efetividade das intervenções breves. A maioria dos artigos da amostra indicou que as IBs são efetivas, com exceção de três artigos, ou porque os pacientes já estavam internados e conseqüentemente abstinentes ao álcool, ou não surtiram efeito em pacientes com comorbidades ou porque a estratégia teve o mesmo efeito que o aconselhamento padrão. Alguns autores dos trabalhos estudados afirmam que as IBs reduzem significativamente o consumo excessivo de álcool, causa impacto positivo e imediato

Quadro 3 - Distribuição dos resultados relacionados à efetividade da intervenção breve nos estudos de revisão bibliográfica nos bancos de dados Lilacs, Scielo e Medline entre outubro de 1997 e janeiro de 2010.

\begin{tabular}{|c|c|}
\hline $\begin{array}{c}\text { Artigo } \\
\text { Número de } \\
\text { Referência }\end{array}$ & Efetividade \\
\hline 08 & A IB reduziu significativamente episódios de bebedeira em bebedores padrão Binge \\
\hline 09 & A IB reduz o consumo de álcool por mais de 12 semanas quando associada ao uso de naltroxena \\
\hline 10 & Tanto a terapia em grupo, quanto a terapia individual são efetivas para a redução do consumo de álcool \\
\hline 11 & Com uma única sessão de IB, os participantes reduziram significativamente os níveis de consumo \\
\hline 12 & Duas sessões de EM são suficientes para que os pacientes fiquem motivados e mudem sua conduta com relação ao álcool \\
\hline 13 & Diminuiu significativamente o consumo de álcool e o número de bebedores de alto risco \\
\hline 14 & Redução do consumo de álcool e da incidência de doenças \\
\hline 15 & $\begin{array}{l}\text { A IB tem impacto benéfico em população de bebedores graves com fácil aplicação em serviços de atenção primária. Pacientes } \\
\text { submetidos à EM tem maior compromisso com o tratamento }\end{array}$ \\
\hline 16 & Menor consumo de drogas, diminuição do estágio motivacional de pré-contemplação \\
\hline 17 & Redução significativa do número de bebidas consumidas, número de dias de consumo e número de dias bebendo pesado \\
\hline 18 & Aconselhamento foi considerado a melhor técnica para o consumo de álcool \\
\hline 19 & Pacientes reduziram significativamente o total de bebidas ingeridas e o número de dias bebendo \\
\hline 20 & As IBs não surtiram efeito em pacientes com comorbidades \\
\hline 21 & A IB for efetiva, diminuindo os níveis de consumo \\
\hline 22 & A EM e o aconselhamento breve são efetivos. Porém a EM reduz o consumo por até 12 semanas \\
\hline 23 & Quanto maior o número de intervenções, maior é a diminuição do consumo \\
\hline 24 & A IB não tem maior efeito que o aconselhamento padrão \\
\hline 25 & A IB não foi eficaz porque os pacientes estavam internados e já abstinentes de álcool \\
\hline 26 & A alta efetividade da IB levou á diminuição do colesterol e dos triglicérides nos bebedores de risco \\
\hline 27 & Demonstraram reduções no consumo de álcool e na frequência do consumo excesivo \\
\hline 28 & Uma única sessão reduziu o consumo de álcool e reduziu a intensidade dos problemas e o comportamento de risco \\
\hline 29 & Houve uma diminuição notável no consumo de álcool médio diário \\
\hline 30 & A IB reduz o consumo de álcool em idosos de forma semelhante à populações jovens \\
\hline 31 & A IB foi eficaz tanto para pacientes com consumo excessivo de álcool, quanto para aqueles com síndrome de dependência alcoólica \\
\hline 32 & A IB foi efetiva para a população aplicada \\
\hline 33 & Reduz significativamente o consumo de álcool \\
\hline 34 & O aconselhamento foi associado com a redução do consumo, e a rotina do ambulatório pode reduzir a eficácia da IB \\
\hline 35 & A IB é associada com a redução contínua do consumo de álcool em mulheres em idade fértil \\
\hline 36 & Houve redução do número de bebedores excessivos \\
\hline 37 & A intervenção foi claramente eficaz para bebedores crônicos \\
\hline
\end{tabular}


nos usuários e que tais estratégias são mais eficazes no campo da atenção primária.

\section{DISCUSSÃO}

Estudos científicos relacionados à aplicação de intervenções breves no tratamento de pacientes bebedores de álcool começam a ser realizados intensamente a partir de 2003. O interesse dos pesquisadores por essa área clínica é vasto, uma vez que os resultados dessa terapia são motivadores para pacientes e profissionais.

São reconhecidas as indicações das IBs para a redução de índices de morbimortalidade, sendo que a mortalidade pode ser reduzida em $23 \%$ e $36 \%$ entre bebedores pesados, como foi encontrado em um estudo realizado em $2006^{(38)}$. Este resultado tem relevância clínica e psicossocial, pois podem prevenir problemas causados pelo uso excessivo de álcool, como aparecimento de doenças cardiovasculares, acidentes de trânsito; e gerar mudanças comportamentais nos indivíduos, o que impacta positiva e diretamente nas taxas de mortalidade.

A técnica da IB pode ser aplicada por profissionais de várias formações, desde que tenham recebido um breve treinamento. Desde simples recomendações para redução do consumo, fornecidas por um profissional (assistente social, enfermeira ou técnica de enfermagem), até uma série de recursos oferecidos em um programa estruturado de tratamento podem ser empregados. Como exemplificado em 2004 por Marques et.al., um médico do Programa de Saúde da Família, pode incorporar em sua consulta de rotina um espaço para identificação de pacientes potenciais para o uso excessivo de álcool, incluindo a aplicação da IB durante a entrevista a ser realizada com o paciente ${ }^{(40)}$. Apesar de existir a negação e resistência em alguns pacientes, os bebedores de risco são, na maioria das vezes cooperativos. A experiência obtida no estudo realizado pela OMS em 2001 demonstra que os pacientes são cooperativos e a maioria deles aprecia que os profissionais de saúde mostrem a interação do álcool com a saúde ${ }^{(41)}$.

Uma importante ferramenta para a definição de estratégias de prevenção são os instrumentos de rastreamento (screening). Eles possibilitam a introdução de procedimentos de intervenção breve e motivam o paciente a mudar seu comportamento. O principal deles para o uso de álcool é o AUDIT (Alcohol Use Disorders Identification Test), de fácil aplicação e com validação universal. Quando associado à intervenção breve, o AUDIT facilita a aproximação inicial e permite um feedback positivo para o paciente, possibilitando assim, a identificação dos níveis de consumo do usuário em questão, o que facilita a definição de estratégias de acordo com o resultado do teste. Usuários identificados no teste como bebedores pesados podem se beneficiar do uso do teste, pois estratégias de IBs podem ser postas em prática antes que estes sofram as complicações mais graves e evoluam para a dependência de álcool ${ }^{(43)}$.

Nesta revisão literária, somente cinco estudos utilizaram o instrumento AUDIT. Os artigos predominantemente não apresentaram instrumentos de rastreamento; dentre os 30 artigos, mais de $50 \%$ não indicaram qualquer ferramenta de suporte à intervenção. Assim o presente estudo tem algumas limitações como não identificar um instrumento considerado padrão-ouro para o rastreamento de consumo do álcool.

A população alvo da aplicação das IBs é diversificada, o que demonstra que os efeitos do uso abusivo de álcool incidem diretamente na vida de indivíduos, independente de sua faixa etária. O estudo realizado por De Micheli et.al., 2004, comprova que a IB é efetiva na redução do consumo de substâncias, além de diminuir a intensidade dos problemas e dos comportamentos de risco ${ }^{(28)}$.

Algumas publicações científicas encontradas selecionaram uma população amostral adolescente ${ }^{(10,13,16,28)}$. Considerando que o contato ou a experimentação de substâncias psicoativas parece ser inevitável para a maioria das pessoas desse grupo etário, a orientação preventiva parece minimizar as conseqüências observadas em jovens que cumprem medidas protetivas, por exemplo, conforme verificado em população

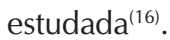

De acordo com os artigos avaliados, os homens apresentam maior consumo de álcool e dessa forma, maior dependência. Do total de 30 artigos da amostra, sete destes tinham homens como população total estudada ou predominante. Essas pessoas, em sua maioria, foram abordadas no serviço de atenção primária à saúde, salvo uma amostra específica de componentes do corpo de bombeiros de uma cidade mineira ${ }^{(21)}$. Essa atenção particular a uma classe trabalhadora demonstra a necessidade de abordagem, avaliação e encaminhamento de possíveis bebedores de risco para os serviços especializados no tratamento de álcool.

Mesmo não sendo predominante nos estudos relacionados ao consumo de álcool, a população feminina teve uma representatividade significativa, já que na amostra final desta revisão, quatro artigos abordaram a temática no universo feminino. O consumo de álcool entre mulheres, principalmente entre adolescentes e mulheres com idades entre 40 e 55 anos mostra-se ascendente na sociedade brasileira. Bem como na amostra masculina, existe predominância de abordagem de usuárias no serviço primário, no entanto, alguns estudos mostraram que é possível a identificação de homens e mulheres que fazem uso excessivo de álcool em outros ambientes, além da atenção primária.

Estudo aplicou as IBs em clínicas obstétricas de serviços de atenção primária que realizavam o acompanhamento pré-natal da gestante. Uma minuciosa entrevista era realizada com a parturiente, a fim de se identificar possíveis riscos à gestação. Comprovou-se que após seis meses de aplicação das IBs houve uma significativa redução no número de bebidas consumidas, no número de dias de consumo e no número de dias bebendo pesado ${ }^{(17)}$.

Outro segmento etário abordado por dois artigos de nosso estudo foi o de idosos. Nesses, os problemas relacionados ao álcool não são divulgados e freqüentemente passam despercebidos aos olhos dos profissionais de saúde. Nos usuários em que o uso abusivo de álcool se desenvolveu a partir dos 50 anos, as perdas e o isolamento que acompanham o envelhecimento se mostraram como fatores que, muitas vezes estão associados com o incídio dos problemas relacionados ao beber pesadamente. Nos 
estudos, os autores indicaram que as IBs conseguiram reduções significativas no consumo e na freqüência do consumo excessivo de álcool e que estas estratégias se mostram tão efetivas na população idosa, quanto em jovens ${ }^{(27,30)}$.

O serviço de atenção primária à saúde é o primeiro contato do indivíduo, da família e da comunidade com o sistema de saúde. É o melhor ambiente para a aplicação de medidas preventivas, pois o estigma com o alcoolista é menor e conseqüentemente também será menor a sua resistência à abordagem e orientação a respeito do problema. A atenção primária é caracterizada por uma boa relação de custo e efeito, pois consegue atingir um número amplo de pessoas e tem oportunidade de intervir antes que o padrão de uso de álcool provoque danos graves à saúde do usuário. Vale ressaltar que nos municípios de pequeno porte no Brasil, o impacto destas estratégias pode ser ainda maior, uma vez que a atenção primária é a principal, senão a única, forma de oferta de serviços públicos de saúde.

O uso excessivo de álcool aumenta a incidência de muitas doenças, com perdas associadas à qualidade de vida e, conseqüentemente, à expectativa de vida. Uma ação preventiva realizada eficazmente aumenta a expectativa de vida da população, e reduz os custos sociais e de cuidados de saúde, como apresentado por estudo que confirma a relação custobenefício das IBs e sugere a implementação das mesmas em serviços de atenção primária à saúde, como excelente estratégia oportunista de rastreio de usuários, já que esses estão na sala de espera dos serviços de saúde ${ }^{(14)}$. Também se pode observar os benefícios que as IBs produzem diretamente à saúde humana ao se analisar estudo ${ }^{(26)}$ realizado porem 2005 com 78 bebedores de risco, que comprovou que após um ano de utilização das Ibs como estratégia para tratamento, verificou-se reduções nos níveis de colesterol, triglicérides, Gama-glutamil transferase (GGT) e Volume corpuscular médio (VCM).

Em 2008 uma pesquisa realizada durante seis meses com aproximadamente 326 indivíduos dependentes de álcool e não dependentes, comprovou que ambos os grupos se beneficiaram das estratégias de intervenção breve, atingindo níveis significativos de redução do total de bebidas ingeridas e o número de dias de consumo ${ }^{(19)}$.

Analisando os artigos desta revisão, contatou-se que a intervenção breve foi eficaz e reduziu significativamente os episódios de bebedeira, obtendo resultados eficazes com apenas uma sessão de $\mathrm{IB}^{(11,28)}$. Em apenas três artigos da amostra a IB não foi efetiva: os pacientes com comorbidades não foram beneficiados com a técnica ${ }^{(20)}$; autores obtiveram melhores resultados de tratamento quando empregaram o aconselhamento padrão, ou seja, a prática de IB não surtiu efeitos superiores à estratégia já empregada; a IB não foi eficaz porque os pacientes já estavam internados, e, consequentemente, abstinentes de álcool ${ }^{(25)}$.

De uma forma geral, a intervenção breve apresenta um enfoque educativo e motivacional para pessoas, às quais se intenciona a redução do uso de álcool. Entretanto, existem dificuldades para a implementação da intervenção na rotina dos serviços de atenção primária à saúde, como a falta de engajamento dos profissionais de saúde e a descrença acerca da possibilidade de melhora dos usuários em relação aos problemas associados ao uso abusivo de álcool ${ }^{(42)}$.

\section{CONSIDERAÇÕES FINAIS}

Dentre os 30 artigos selecionados para o estudo sistemático da literatura, entre os anos de 1997 a 2010, que versavam acerca da IB como estratégia empregada em serviços da atenção primária, para a redução do consumo abusivo de álcool, outros estudos publicados utilizaram teste de rastreamento de uso abusivo de álcool, como o AUDIT, o que dificulta saber a quantidade de álcool que a pessoa consumia, e conseqüentemente, repercute na confiabilidade das intervenções breves, quanto a serem efetivas ou não.

Além disso, a não caracterização dos participantes em todos os estudos impossibilitou estabelecer, em todos eles, o gênero e a faixa etária mais suscetíveis ao consumo excessivo de álcool, e também, em qual desses grupos as IBs são mais eficazes.

A falta de conscientização dos profissionais de saúde acerca do alcoolismo, como sendo verdadeiramente uma questão de saúde pública, os leva a estigmatizar os consumidores abusivos e a não se envolver com estratégias de enfrentamento como as IBs, em sua rotina profissional.

Os resultados encontrados mostraram que o local mais adequado para a implantação destas estratégias o serviço de atenção primária. Porém, para o maior sucesso da técnica ressalta-se a importância do engajamento dos coordenadores das unidades básicas de saúde (UBS) na implementação dessa proposta, estimulando e proporcionando a capacitação de seus funcionários. Deve-se levar em consideração que qualquer profissional, de nível superior, da área da saúde tem habilidades suficientes para atingir bons resultados na aplicação das IBs, desde que esteja capacitado.

Ademais, pode-se aproveitar o potencial que os agentes comunitários têm em transitar seus territórios, para que os mesmos possam identificar os consumidores abusivos nos espaços comunitários, contribuindo para que os profissionais tenham acesso a eles e possam aplicar as estratégias de IBs.

Alguns estudos preconizam que as IBs devem ser direcionadas apenas aos bebedores com comportamento de risco ou consumo nocivo, pois esses ainda não se apresentam com sinais de dependência. Outros autores afirmam que os bebedores já diagnosticados dependentes se beneficiam também do uso destas estratégias, considerando que esse seria o primeiro passo, pois as IBs se caracterizam como políticas motivacionais e não invasivas.

As autoras acreditam que as IBs devam ser incorporadas nas políticas públicas de saúde em atenção aos usuários de álcool e outras drogas, como estratégia para o enfrentamento do consumo abusivo do álcool, antes que esse chegue à dependência química. Dessa forma, tais estratégias seriam financiadas pelo Ministério da Saúde e todos os profissionais e equipes de saúde da atenção básica seriam capacitados para realizá-las.

Por fim, as autoras fazem uma provocação aos enfermeiros que atuam no cuidado em saúde mental, para que publiquem artigos relatando suas experiências com IBs, uma vez que nos artigos estudados, alguns dos estudos foram realizados por enfermeiros (a coleta de dados), mas publicados por outras categorias profissionais. 


\section{REFERÊNCIAS}

1. Centro de Informações sobre Saúde e Álcool [homepage na internet]. [acesso em 24 maio 2011]. Diponível em: http://www.cisa.org.br/

2. Marques ACPR. O uso do álcool e a evolução do conceito de dependência de álcool e outras drogas e tratamento. Rev IMESC 2001;3:73-86.

3. Segre M, Ferraz FC. O conceito de saúde. Rev Saúde Pública 1997;31(5):538-542.

4. Scliar M. História do conceito de saúde. Physis (Rio J.) 2007; 17(1):29-41.

5. De Micheli D, Fisberg M, Formigoni MLOS.Estudo da efetividade da intervenção breve para o uso de álcool e outras drogas em adolescentes atendidos num serviço de assistência primária à saúde. Rev Assoc Med Bras 2004;50(3):305-313.

6. Marques ACPR, Furtado EF. Intervenções breves para problemas relacionados ao álcool. Rev Bras Psiquiatr 2004;26(Suppl.1):28-32.

7. Ronzani TM, Ribeiro MS, Amaral MB, Formigoni MLOS. Implantação de rotinas de rastreamento do uso de risco de álcool e de uma intervenção breve na atenção primária à saúde: dificuldades a serem superadas. Cad Saúde Pública 2005;21(3):852-861.

8. Rubio G, Jimenez- Arriero MA, Martinez I, Ponce G, Palomo T. Efficacy of physician- delivered brief couseling intervention for binge drinkers. Am J Med 2010;123(1):72-8.

9. Castro LA, Laranjeira R. Ensaio clínico duplo- cego randomizado e placebo- controlado com naltrexona e intervenção breve no tratamento ambulatorial de dependência de álcool. J Bras Psquiatr 2009;58(2):79-85.

10. Flórez Alarcón L, Gantiva Diaz YCA. Terapias motivacionales breves: diferencias entre la aplicación individual y grupal para la moderación del consumo de alcohol y de las variables psicológicas asociadas al cambio. Acta Colomb Psicol 2009;12(1):13-26.

11. Ockene JK, Reed GW, Reiff-Hekking S. Brief patient- centered clinician- delivered counseling for high- risk drinking : 4-year results. Ann Behav Med 2009;37(3):335- 342.

12. Stein LA, Minugh PA, Longobaugh R, Wirtz P, Baird J, Nirenberg TD, et al. Readiness to Change as a Mediator of the Effect of a Brief Motivational Intervention on Posttreatment alcohol-related consequences of injured emergency department hazardous drinkers . Psychol Addict Behav 2009;23(2):185-195.

13. Schaus JF, Sole ML, Mc Cory TP, Mullet N, O`Brien MC. Alcohol screening and brief intervention in a college student health center a randomized controlled trial. J Stud Alcohol Drugs 2009;(Suppl 16): 131-141.

14. Tariq L, Van den Berg M, Hoogenveen RT, Van Baal PH. Cost- effectiveness of an opportunistic screening programme and brief intervention for excessive alcohol use in primary care. PLos ONE 2009;4(5):e5696.

15. Oliveira MS, Andreta I, Rigoni MS, Szpscynski KPR. A entrevista motivacional com alcoolistas: um estudo longitudinal. Psicol Reflex Crít 2008;21(2):261-266.

16. Andretta I, Oliveira MS. Efeitos da entrevista motivacional em adolescentes infratores. Estud Psicol (Campinas) 2008;25(1):45-53.

17. 17. Fleming MF, Lund MR, Wilton G, Landry M, Scheets $D$. The healthy moms study: the efficacy of brief alcohol intervention in postpartum women. Alcohol Clin Exp Res 2008;32(9):1600-1606.

18. Dent AW, Weeland TI, Philips GA, Lee NK. Opportunistic screening and clinician-delivered brief intervention for high- risk alcohol use among emergency department attendees: a randomized controlled trial. Emerg Med Australas $2008 ; 20(2): 121-8$

19. Guth S, Lindberg AS, Badger GI, Thomas CS, Rose GL, Helzer JE. Brief intervention in alcohol-dependent versus nondependent individuals. J Stud Alcohol Drugs 2008;69(2).

20. Grotheus JM, Beschof G, Runhardt S, Meyer C, John U, Rumpf HJ. Effectiveness of brief alcohol intervention for general practice patients with problematic drinking behavior and comorbid anxiety or depressive disorders. Drug Alcohol Depend 2008; 94(1-3):214-20.

21. Batista AG, Lourenço LM, Formigoni MLOS, Ronzani TM, Rodrigues TP. Estratégias de rastreamento e intervenções breves para problemas relacionados ao abuso de álcool entre bombeiros. Estud Psicol 2007;12(3):285-290.

22. Ball SA, Martino S, Nich C, Frankforter TL, Van Horn $D$, Crits-Cristoph $P$, et al. Site matters: multisite randomized trial of motivation enhancemet therapy in community drug abuse clinics. J Consult Clin Psychol 2007;75(4):556-567.

23. Brown RL, Sainders LA, Bobula JÁ, Mundt MP, Koch PE. Randomized- controlled trial of a telephone and mail intervention for alcohol use disorders: three- month drinking outcomes. Alcohol Clin Exp Res 2007;31(8):1372-9.

24. Lock CA, Kaner E, Heather N, Daughty J, Crawshaw A, MC Namee $P$, et al. Effectiveness of nurse- led brief alcohol intervention: a cluster randomized controlled trial. J Adv Nurs 54(4):426-39.

25. Laranjeira R, Payá R, Turisco J, Gomes LCS, Dunn J, Fugli NB. Motivação para a mudança do comportamento de beber: diferenças entre pacientes usuários de álcool do ambulatório de gastroenterologia e do serviço especializado no tratamento do alcoolismo. São Paulo Med J 2005;123(5):223-228.

26. López- Marina V, Pizarro Romero G, Alcolea Garcia R, Beato Fernández P, Galindo Montané E, Montella Jordana N. Evaluación Del cribado y la efectividad de uma intervención breve en bebedores de riesgo atendidos en consultas de atención primaria. Aten Primaria 36(5):261-268.

27. Mundt MP, French MT, Roeobuck MC, Manwell LB, Barry KL. Brief physician advice for problem drinking among older adults: an economic analysis of costs and benefits. J Stud Alcohol 2005;66(3):389-94. 
28. De Micheli D, Fisburg M, Formigoni MLOS. Estudo da efetividade da intervenção breve para o uso de álcool e outras drogas em adolescentes atendidos num serviço de assistência primária a saúde. Rev Assoc Méd Bras 2004;50(3)355-13.

29. Lieber CS, Weiss DG, Grosz Mann R, Paronetto F, Schenker S. Veterans affairs cooperative study of polyenylphosphatidylcholine in alcoholic liver disease: effects on drinking behavior by nurse/ physician teams. Alcohol Clin Exp Res 2003;27(11):1757-1764.

30. Gordon AJ, Conigliaro J, Maisto SA, McNeil M, Kraemer $K L$, Kelly ME. Comparison of consumption effects of brief interventions for hazardous drinking elderly. Subst Use Misuse 2003;38(8):1017-35.

31. Fernández Gárcia JA, Ruiz Moral R, Pérula de Torres LA, Campos Sánchez L, Lora Cerezo N, Martinez de la Iglesia J. Efectividad Del consejo médico a pacientes alcohólicos y bebedores excesivos atendidos en consultas de atención primaria. Aten primaria 2003;31(3):146-53.

32. Kaner EF, Heather N, Brodie J, Lock CA, Mc Avoy BR. Patient and practitioner characteristics predict brief alcohol intervention in primary care. $\mathrm{Br} \mathrm{J}$ Gen Pract 2001;51(471):822-827.

33. Aaalto $M$, Seppä K, Mattila $P$, Mustonen $H$, Ruuth $K$, Hyvärinen $\mathrm{H}$, et al. Brief intervention for male heavy drinkers in routine general practice: a three-year randomized controlled study. Alcohol alcohol 2001;36(3):224-230.

34. Aaalto M, Saksanen R, Laine P, Forsström R, Kiviluoto M, Seppä K, et al. Brief intervention for female heavy drinkers in routine general practice: a three-year randomized controlled study. Alcohol Clin exp Res 200;24(11):1680-6.

35. Manwell LB, Fleming MF, Mundt MP, Stauflacher EA, Barry KL. Treatment of problem alcohol use in women of childbearing age: results of a brief intervention trial.
Alcohol Clin Exp Res 2000;24(10):1517-24.

36. Córdoba R, Delgado MT, Pico V, Altisent R, Fores D, Monreal $\mathrm{A}$, et al. Effectiveness of brief intervention on non- dependent alcohol drinkers (EBIAL): a Spanish multicentre study. J Fam Pract 1998;15(6):562-568.

37. Fernández San Martín MI, Bermejo Cajá CJ, Alonso Pérez $M$, Herreros Taberneros B, Nieto Candenas $M$, Novoa $A$, et al. Effectiveness of brief medical counseling to reduce drinker`s alcohol consumption. Aten Primaria 1997;19(3):127-32.

38. Fontes A, Figlie NB, Laranjeira R. O comportamento de beber entre dependentes de álcool: estudo de seguimento. Rev Psiquiatr Clín 2006;(33):6:304-312.

39. Magnabosco, MB, Formigoni MLOS, Ronzani TM. Avaliação dos padrões de uso de álcool em usuários de serviços de Atenção Primária à Saúde de Juiz de Fora e Rio Pomba (MG). Rev Bras Epidemiol 2007;10(4):637-647.

40. Marques ACPR, Furtado F. Intervenções breves para problemas relacionados ao álcool. Rev Bras Psiquiatr 2004;26(Suppl.1):28-32.

41. Organización Mundial de la Salud. Intervención breve para el consumo de riesgo y prejudicial de alcohol: un manual para la utilización en atención primaria. Valencia: Conselleria de Benestar Social, Generalitat Valenciana; 2001.

42. Ronzani, TM, Ribeiro MS, Amaral MB, Formigoni MLOS. Implantação de rotinas de rastreamento do uso de risco de álcool e de uma intervenção breve na atenção primária à saúde: dificuldades a serem superadas. Cad Saúde Pública 2005;21(3):852-861.

43. Figlie NB, Pillon SC, Dunn J, Laranjeira R. The frequency of smoking and problem drinking among general hospital inpatients in Brazil - using the AUDIT and Fagerström questionnaires. Sao Paulo Med J 2000;118(5):139-143. 